\title{
Diensthabende
}

\section{Echte Helden}

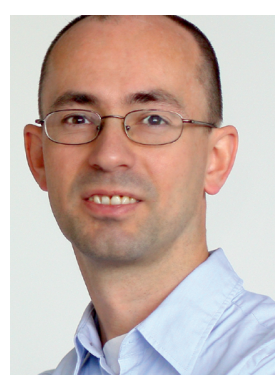

Sind Notärzte Helden? Oberflächlich betrachtet hat der Job ja schon was von Superman und Superwoman: In Momenten größter Not auftauchen, Problem lösen, abhauen. Schaut man genauer hin, erkennt man „kleine“ Unterschiede: Das fängt schon damit an, dass kein Notarzt als alleiniger „Vollstrecker“ agiert. Zwar trifft er die Entscheidungen, doch ohne sein Team wäre er aufgeschmissen. Zudem hält die Notfallmedizin viele Algorithmen und Leitlinien bereit, an denen er sich zu orientieren hat. Für kreative Lösungen à la Bat- oder Spiderman bleibt da wenig Raum. Und auch das rasante Auftauchen am Einsatzort ist keine Leistung der Notärzte. Dass in Deutschland im Schnitt ca. zehn Minuten nach jedem Notruf der Rettungsdienst vor der Tür steht, ist vor allem dem dicht gewebten Netz der Rettungswachen zu verdanken. Bleibt da noch was übrig vom Heldenimage? Auf jeden Fall! Schon allein, weil Notärzte den Mut haben, sich tagtäglich in Situationen zu begeben, bei denen sie nicht mit Sicherheit wissen, was sie am Ende erwartet. Wenn du so ein „Held vom Dienst“ werden möchtest, helfen dir zwei Artikel in diesem Heft: Auf S. 20 erklären wir, wie die Ausbildung abläuft. Und auf S. 34 geben wir eine Einführung in eine der wichtigsten „Skills“, die du in diesem Job brauchst - die Intubation.

Herzlichst

euer Dieter Schmid

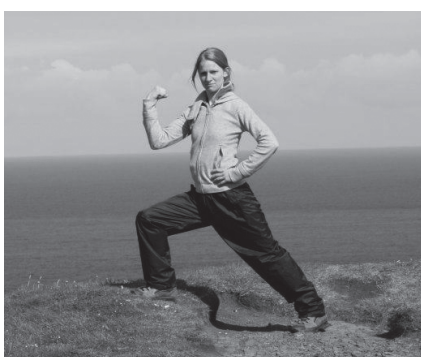

Anna N. Wolter

machte bereits an der irischen Küste Bekanntschaft mit steilen Felsklippen. Als Nächstes stehen Norwegens Fjorde auf dem Programm. Skandinavien ist aber nicht nur ein tolles Reiseland es bietet auch hervorragende Arbeitsbedingungen für Ärzte, wovon Anna in ihrem Artikel berichtet (S. 14).

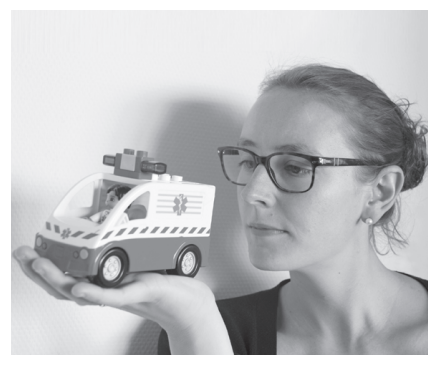

\section{Sarah Hölscher}

findet Notallmedizin spannend - ist aber gottfroh, dass sie selbst noch nie einen Notarzt rufen musste. Für Via medici begab sie sich jetzt zu den „Rotjacken“ und war überrascht, wie unaufgeregt die Retter trotz zwischenzeitlicher Adrenalinduschen agieren (S. 20).

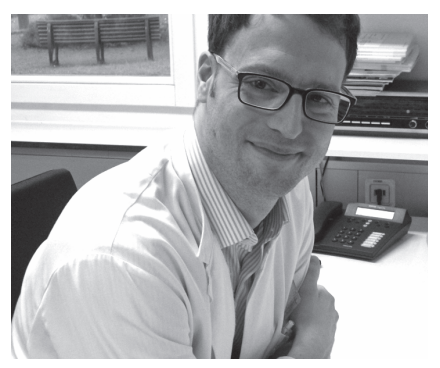

Dr. Berend Malchow begegnet als Facharzt für Psychiatrie die ganze Palette an psychischen Störungen. Besonderes spannend findet er die Schizophrenie mit ihren oft bunten Wahnsymptomen. Wie eine akute schizophrene Psychose erkannt und behandelt werden kann, erklärt er in diesem Heft (S. 46). 